\title{
Traumatic brain injury of childhood
}

\author{
Abdul $\mathrm{H}$ ameed \\ D ean $\mathrm{N}$ eurosciences / Professor of $\mathrm{N}$ eurosurgery, Fatima Jinnah M edical U niversity/Sir G anga Ram H ospital L ahore. \\ Email: abdulhameedns@gmail.com
}

It is a common observation, and very unfortunate one, that only the driver wears or "bears" helmets on motorbikes. N one of the other passengers, especially children, are supposed to be protected against head injury. The same callous attitude is observed in cars where children are sometimes sitting in the lap of the front seater without tightening with the seat belt. $\mathrm{K}$ ids are thrown in the air to recatch them for pleasure or shaken severely. At times intentionally or unintentionally abused and hit or slapped on head or face without understanding how much harm is posed to them psychologically and physically. "Children are not mini-adults." Their unique unprotected anatomy and premature physiology make them vulnerable to catastrophic permanent damage and even death.

T raumatic brain injury ( $\mathrm{T} B \mathrm{~B})$ frequently occurs in young people. It remains the most common cause of disability and death between the ages 1 and $45 .{ }^{1} \mathrm{~T}$ BI cases are increasing rapidly in Pakistan with the increase in population and poor domestic, road, and sports safety considerations. ${ }^{1,2}$ Falls from height remain on top while reviewing the modes of injury followed by road traffic accidents. Child abuse is increasingly observed important reason for a head injury, especially in infants and young children, as they are more vulnerable and dependent on adults. ${ }^{2}$ Abusive head trauma (AHT) is unfortunately common in children up to two years. These children may appear with drowsiness, delayed milestones, seizures, and intracranial traumas of different stages of healing. Other associated injuries may be retinal bleeds, multiple rib fractures, and long bone fractures of different stages of recovery. Child abuse should be promptly identified and appropriately reported.

Children are different from adults and more vulnerable to traumatic brain injuries because of their proportionately larger head, low blood circulating volume, and thin osteo-fibrous non-rigid skull. M ore watery and less myelinated brain, non-aerated paranasal sinuses, and less CSF cushion to protect shaking and damage. C hildren are also vulnerable to $\mathrm{T} B \mathrm{BI}$ during the birth process. During normal delivery and especially

D 0I: https://doi.org/10.37018/Z N CR 2683 difficult instrument-assisted births neonates are left with various types of intracranial hemorrhages. Cephalic and subgaleal hematomas are not uncommonly seen. T his is due to increased flexibility or decreased rigidity of the neonatal skull. This increased elasticity of premature skull caused some degree of skull deformation in small children when they are shacked. The irregular base of the skull, especially in the anterior and middle cranial fossa, and the absence of adequate CSF cushion and watery consistency of the brain make it vulnerable to "shaken baby syndrome." $\mathrm{W}$ hen a child is severely shacked, stretching and shearing forces may tear vessels and cause contusions in brain parenchyma. It may end up in subdural hematomas and intraparenchymal contusions.

$\mathrm{H}$ ead size in the pediatric age group is proportionately larger than the body, making the head a bigger target for trauma. A thin neck also accompanies this with weak neck muscles, and it increases the chances of neck trauma and cervical spinal cord injury in children. It is mandatory to inspect and rule out any spinal injury with head trauma. Children have not yet developed paranasal air sinuses that could absorb force of direct impact on the skull and hence the brain. It makes the brain less protected from trauma. Children also have less circulating volume, so lacerating scalp wounds, contrary to adults, may be cause of hypovolemic shock and death in children.

Skull fractures and the resulting severity of $\mathrm{T} B \mathrm{~B}$ in children are similar to in adults. ${ }^{1-3} \mathrm{C}$ hild may come with huge linear fractures spanning over parietal, occipital, temporal, and frontal bones. T hese may or may not be associated with dural tears and CSF leaks. Most fractures may not need surgical treatment. $T$ here are two types of fractures specific for the early age group. A greenstick ping pong ball fracture occurs when a parietal eminence strikes over or is hit upon. It usually is associated with falls from the bed over a hard surface. Spontaneous resolution is observed in early neonates, but in toddlers, it may need surgical elevation if untreated is a potential cause of seizure activity of the brain in the future. Growing skull fracture of neonates and children younger than two years is earlier a linear fracture that grows due to pulsatile leptomeningeal or brain herniation through free edges of fracture and 
separating these edges apart. It should be treated surgically. Epidural hematomas in children are primarily venous sinus hemorrhages when fracture line runs over and tears the venous sinuses. Arterial causes of epidural hematoma are also possible. Indications for surgery in symptomatic patients are the same as in adults. M ost non-symptomatic hematomas may resolve spontaneously by seepage through overlying skull fractures and absorption in subgaleal space. ${ }^{3}$

Goals and means of treatment of traumatic brain injury are similar in children as in adults. ${ }^{3,4}$ Primary goal of treatment is to identify primary brain insults and prevent their conversion into secondary brain injure. Secondary brain injury is a result of an ongoing increase in brain oedema and reduction in cerebral perfusion. Resulting brain ischemia may cause death or irreversible mental damage. This can be achieved, stepwise, by elevation of the head by 30 degrees to facilitate C SF and venous drainage out of intracranial compartment while keeping airway patent, oxygenation, use of osmotic diuretics, external ventricular drainage of CSF, evacuation of intracranial hematomas, elective ventilation and barbiturate coma, hyperventilation with targets of $\mathrm{CO}_{2}$ between 30 and 35 , decompressive craniotomies and hypothermia as a last resort. The goal of all these measures is to decrease intracranial pressure and increase cerebral perfusion. Patients with a low Glasgow Coma Scale at arrival, like 3 or 4, have very few chances of surviving one year after trauma. G eneral school-going toddlers with mild traumatic brain injury have good outcomes. These children may need prolonged psychological assistance to transition from preschool to school life..$^{1-4}$

Particular attention should be given to children who tolerate abusive head trauma. These children are under constant torture from their caregivers. So prompt identification and reporting to the concerned government department is the health caregiver's moral and legal obligation. T ransport rules should be made and implemented for safety measures in motorbikes and an unrestrained car traveling.

\section{REFERENCES}

1. Adamson M M, Shakil S, Sultana $T, H$ asan MA, M ubarak F, Enam SA, et al. Brain injury and dementia in Pakistan: Current perspectives. Front N eurol. 2020; 11:299. DOI: 10.3389/fneur.2020. 00299

2. Asif M, Rehman W A, Serwar S, Y ounas H , Y ounas F. Pediatric head injury: A study of 120 cases. Pak J of N eurol Surg. 2021; 25(2):180-6. D Ol:10.36552/pjns.v25i2.557

3. Araki T, Yokota H, M orita A. Pediatric traumatic brain injury: $C$ haracteristic features, diagnosis, and management. $N$ eurol Med Chir (T okyo). 2017: 57, 82-93. DOI: 10.2176/nmc.ra. 2016-0191

4. Derakhshanfar H, Pourbakhtyaran E, Rahimi S, Sayyah S, Soltantooyeh Z, Karbasian F. Clinical guidelines for traumatic brain injuries in children and boys. E ur J T ransl M yol. 2020; 30 (1): 159-170. 